\title{
On Germs of Differentiable Functions in Two Variables
}

By

\author{
Masahiro Shiota
}

\section{§1. Introduction}

In [4] we have shown some sufficient conditions for a germ (of a differentiable function in two variables) to be transformed into an analytic one or a polynomial through a change of coordinates. Here we shall refine the result above and find a necessary and sufficient condition.

We denote respectively by $\mathcal{O}, \mathscr{E}$ the rings of germs at 0 in $\mathbb{R}^{2}$ of real analytic and $C^{\circ}$-functions, and by $\mathscr{F}$ the ring of formal power series in 2 indeterminates over $\mathbb{R}$. If $A$ is one of the above rings, let $\mathfrak{m}(A)$ denote the maximal ideal of $A$. Let $T_{a}$ denote the Taylor expansion at $a$. Elements $f, g$ of $\mathscr{E}$ are called equivalent if there exists a local diffeomorphism (of class $C^{\infty}$ ) $\tau$ of $\mathbb{R}^{2}$ around 0 such that $f \circ \tau=g$. If an element $f$ of $\mathfrak{m}(\mathscr{E})$ can be factorized into the following form

$$
f=\prod_{i=1}^{n} f_{i}^{\alpha_{\imath}}
$$

such that $T_{0} f_{i}(\neq 0)$ are prime and that any two of them are relatively prime in $\mathscr{F}$, then we call $f$ factorizable.

Theorem 1. Let $f$ be in $\mathrm{m}(\mathscr{E})$. Then $f$ is equivalent to a non-zero element of $\mathcal{O}$ if and only if $f$ is factorizable.

Theorem 1 has the following corollary.

Corollary. Any element of $\mathrm{m}(\mathscr{E})$ is equivalent to some $f+g$ where $f$ is in $\mathcal{O}$ and where $g$ is in $\mathscr{E}$ and flat (i.e. $T_{0} g=0$ ).

The following is another result. 
Theorem 2. Any element of $\mathcal{O}$ is equivalent to some polynomial one. We note that Theorem 2 is also valid in the complex case.

The author wishes to thank Prof. M. Adachi for his kind advices.

\section{§2. Proofs of Theorems}

For the proof of Theorem 1 we need the following lemmas.

Lemma 1 (Shiota [4]). Let $f, g$ be in $\mathscr{E}$ and $a_{i}(x, t)(i=1,2)$ be germs at $0 \times[0,1]$ in $\mathbb{R}^{2} \times \mathbf{R}$ of $C^{\infty}$-functions. If the conditions

$$
\begin{aligned}
& f(x)-g(x)=\sum_{i=1}^{2} a_{i}(x, t)\left(\frac{\partial f}{\partial x_{i}} t+\frac{\partial g}{\partial x_{i}}(1-t)\right), \\
& a_{i}(0, t)=0
\end{aligned}
$$

are satisfied, then $f$ and $g$ are equivalent.

Lemma 2 (Malgrange [3]). Let $\widetilde{\mathscr{F}}$ be the ring of germs at 0 in $\mathbb{R}^{2}$ of collections of formal power series at each point near 0 in $\mathbb{R}^{2}$. We may regard $\mathscr{E}$ as a subring of $\tilde{\mathscr{F}}$. Let $\mathfrak{p}$ be an ideal in $\mathcal{O}$. Then we have

$$
(p \tilde{\mathscr{F}}) \cap \mathscr{E}=\mathfrak{p} \mathscr{E} \text {. }
$$

Lemma 3 (Shiota [4]). If an element $f$ of $\mathrm{m}(\mathscr{E})$ is factorizable, then there exists a germ $\phi \in \mathscr{E}-\mathfrak{m}(\mathscr{E})$ such that $\phi f$ is equivalent to an element of $\mathcal{O}$.

Lemma 4. Let $f$ be in $\mathfrak{m}(\mathscr{F})$ (resp. $\mathfrak{m}(\mathcal{O})$ ). Then, for some integer $k>0, f \mathfrak{m}^{k}(\mathscr{F})$ (resp. $f \mathfrak{m}^{k}(\mathcal{O})$ ) is contained in the ideal generated by $\frac{\partial f}{\partial x_{i}}$ in $\mathscr{F}($ resp. $\mathcal{O})$.

Proof of Lemma 4. From the fact that $\mathscr{F}$ is faithfully flat over $\mathcal{O}$, it is enough to prove only for $\mathscr{F}$. As $\mathscr{F}$ is a unique factorization ring, there are prime elements $f_{i} \in \mathscr{F}$ such that

$$
f=\prod_{i=1}^{n} f_{i}^{\alpha_{i}}
$$

where $f_{i}, f_{j}(i \neq j)$ are relatively prime. Let $f^{\prime}=\prod_{i=1}^{n} f_{i}^{\alpha_{2}-1}$. Then $\frac{\partial f}{\partial x_{i}}$ are 
divisible by $f^{\prime}$. We can easily see that for each $i$ at least one $\frac{\partial f}{\partial x_{j}} / f^{\prime}$ is not divisible by $f_{i}$. Let $\mathfrak{p}$ be the ideal generated by $\frac{\partial f}{\partial x_{i}} / f^{\prime}$. Let us show now that the height of $\mathfrak{p}$ is two. If its height is one, then $\mathfrak{p}$ is contained in a prime ideal $q$ of height one. According to a result in [5] that

$$
f^{m}=\sum_{i=1}^{2} h_{i} \frac{\partial f}{\partial x_{i}} \text { for some integer } m>0 \text { and } h_{i} \in \mathscr{F}
$$

$\mathfrak{q}$ contains $f$ and hence also $f_{i}$ for some $i$. The height of $\mathfrak{q}$ being one, we have $\mathfrak{q}=f_{i} \mathscr{F}$. This means that $\frac{\partial f}{\partial x_{i}} / f^{\prime}$ are divisible by $f_{i}$; that is a contradiction. Hence the height of $\mathfrak{p}$ is two, and $\mathfrak{p}$ contains $\mathfrak{m}^{k}(\mathscr{F})$ for some integer $k>0$. Therefore the ideal generated by $\frac{\partial f}{\partial x_{i}}$ contains $f^{\prime} \mathfrak{m}^{k}(\mathscr{F})$ and so $f \mathfrak{m}^{k}(\mathscr{F})$.

Proof of Theorem 1. For the necessity of the condition, see [4]. Let us prove its sufficiency. Suppose that an element $f$ of $\mathfrak{m}(\mathscr{E})$ is factorizable. By Lemma 3 we may regard $\phi f$ as in $\mathcal{O}$ for some germ $\phi \in \mathscr{E}-\mathfrak{m}(\mathscr{E})$. Here, for each integer $k>0$, we may choose a germ $\phi$ of the form $\phi=1+\phi$ 。 with $\phi_{\circ}$ in $\mathrm{nt}^{k}(\mathscr{E})$. In fact we have

$$
T_{0} \phi / \phi(0)=1+H_{i}+H_{i+1}+\cdots
$$

for some integer $i>0$ where $H_{i}$ are the homogeneous parts of degree $i$, then we see

$$
\begin{aligned}
& \phi / \phi(0)-1 \in \mathfrak{m}^{i}(\mathscr{E}) \\
& \left(1-H_{i}\right) \phi / \phi(0)-1 \in \mathfrak{m}^{i+1}(\mathscr{E}) .
\end{aligned}
$$

This gives the statement above.

Let $\phi f=g, \phi^{-1}=\psi, \phi=1+\psi_{\text {。 }}$.

When the above $k$ is sufficiently large, from Lemma 4 there exist elements $b_{i}, c_{i, j}$ of $\mathfrak{m}(\mathscr{F}) i, j=1,2$ such that

$$
\begin{aligned}
& T_{0}\left(\psi_{\circ} g\right)=\sum_{i=1}^{2} b_{i} T_{0}\left(\frac{\partial g}{\partial x_{i}}\right) \\
& T_{0}\left(\frac{\partial \psi_{\circ}}{\partial x_{i}} g\right)=\sum_{j=1}^{2} c_{i, j} T_{0}\left(\frac{\partial g}{\partial x_{j}}\right) .
\end{aligned}
$$


If $g$ has no multiple factors, Lemma 4 shows that the height of the ideal generated by $\frac{\partial g}{\partial x_{i}}$ in $\mathcal{O}$ is two, and that the set of critical points of $g$ is $\{0\}$. It is shown that for each point $a \neq 0$ near 0 with $g(a)=0$, the germ of $g$ at $a$ is equivalent to $x_{1}$ where $\left(x_{1}, x_{2}\right)$ is a local coordinates around $a$ such that $a=(0,0)$. We remark that exactly one factor of $g$ vanishes at $a$. When $g$ has multiple factors, by the remark above we easily see that for the same point $a$ as the above, the germ of $g$ at $a$ is equivalent to $x_{1}^{\alpha}$ for some integer $\alpha>0$. Because of Lemma 4 we have for some integer $m>0$ and $h_{i} \in \mathcal{O}$

$$
g^{m}=\sum_{i=1}^{2} h_{i} \frac{\partial g}{\partial x_{i}}
$$

This shows that at least one first partial derivative does not vanish at each point $a$ near 0 with $g(a) \neq 0$. From these, $T_{a} g$ is contained in the ideal generated by $T_{a} \frac{\partial g}{\partial x_{i}}$ in $\mathscr{F}$ except when $a=0$. By Lemma 2 there exist elements $\beta_{i}, \gamma_{i, j}$ of $\mathfrak{m}(\mathscr{E}) i, j=1,2$ such that

$$
\begin{aligned}
& \psi_{\circ} g=\sum_{i=1}^{2} \beta_{i} \frac{\partial g}{\partial x_{i}} \\
& \frac{\partial \psi_{\circ}}{\partial x_{i}} g=\sum_{j=1}^{2} \gamma_{i, j} \frac{\partial g}{\partial x_{j}} .
\end{aligned}
$$

We can prove in the same way as in the proof of Proposition 1 in [4] that $f, g$ satisfy the condition in Lemma 1 ; hence $f$ is equivalent to $g$.

Proof of Theorem 2. Let $f$ be an element of $\mathcal{O}$. Since $\mathcal{O}$ is a unique factorization ring, there are prime elements $f_{i} \in \mathcal{O}$ and positive integers $\alpha_{i}$ such that

$$
f=\prod_{i=1}^{n} f_{i}^{\alpha_{i}}
$$

where $f_{i}, f_{j}(i \neq j)$ are relatively prime. Let

$$
g=\prod_{i=1}^{n} f_{i}
$$

and let $\bar{f}_{i}$ be polynomials of degree $k$ which have the same partial 
differential coefficients at 0 up to $k$ order as $f_{i}$ where $k$ is sufficiently large. We know the following fact (Tougeron [5]). Let $h$ be an element of $\mathrm{m}(\mathcal{O})$ without multiple factors. Then there is an integer $k>0$ such that $h$ is equivalent to any element of $\mathcal{O}$ which has the same partial differential coefficients up to $k$ order as $h$. This shows that $g$ is equivalent to $\prod_{i=1}^{n} \bar{f}_{1}$. Hence there exists a local diffeomorphism $\tau$ of $\mathbb{R}^{2}$ around 0 such that $g \circ \tau=\prod_{i=1}^{n} \bar{f}_{i}$. By the uniqueness of the factorization, there exist invertible elements $\psi_{i}$ of $\mathcal{O}$ such that $\phi_{i}\left(f_{i} \circ \tau\right)=\bar{f}_{i^{\prime}}$ where $i^{\prime}$ is not always $i$. Let

$$
\phi=\prod_{i=1}^{n} \psi_{i}^{\alpha_{\iota}} .
$$

Then we see that $\phi(f \circ \tau)$ is a polynomial element, this result corresponds to our Lemma 3 in the differential case. It is enough for the remainder of the proof to proceed in the same way as in the proof of Theorem 1 .

\section{§3. An application}

Let us recall the following concept introduced by Kuo [2].

Definition. An element $f \in \mathscr{F}$ is called $C^{0}$-sufficient in $C^{r}$ if for any two $C^{r}$-realizations $g, g^{\prime}$ of $f$ (i.e. $g, g^{\prime}$ are $C^{r}$-functions defined near 0 and the natural images of $f, g, g^{\prime}$ in $\mathscr{F} / \mathrm{m}^{r+1}(\mathscr{F})$ coincide) there exists a local homeomorphism $\tau$ around 0 such that $g \circ \tau=g^{\prime}$ near 0 .

An element $f \in \mathscr{F}$ is simply called $C^{0}$-sufficient if $f$ is $C^{0}$-sufficient in $C^{r}$ for some $r$.

Theorem 3 (Kuo [2]). An element $f \in \mathscr{F}$ is not $C^{0}$-sufficient if and only if there is a $C^{\infty}$-realization $g$ of $f$ which satisfies the condition that $g$ is divisible by an element of the form $h^{2}$ where $h$ has zeros arbitrary close to 0 . If $f$ is in $\mathcal{O}$ (resp. a germ of a polynomial), we may choose $g, h$ in $\mathcal{O}$ (resp. in the domain of germs of polynomials).

Proof. Theorem 7-2 in [2] treats only the polynomial case but it proof is also valid in the analytic case. For the differentiable case, the necessity of the condition is obvious from our Corollary in $\$ 1$, and its 
sufficiency follows from the converse of the Kuiper-Kuo Theorem in [1] (i.e. if $g$ is a $C^{r}$-realization of a $C^{0}$-sufficient element in $C^{r}$ then $/ \mathrm{grad}$ $\left.g(x)|\geqq c| x\right|^{r-1}$ in a neighbourhood of 0 , with some $c>0$ ).

\section{Theferenter}

[1] Bochnak, J. and Lojasiewicz, S., A converse of the Kuiper-Kuo Theorem, Lecture Notes, in Math. Springer, 192 (1971), 254-261.

[2] Kuo, T.C., The jet space $J^{r}(n, p)$, ibid. 169-177.

[3] Malgrange, B., Ideals of differentiable functions, Oxford University Press, 1966.

[4] Shiota, M., Transformations of germs of differentiable functions through changes of local coordinates, RIMS, Kyoto Univ. 9 (1973), 123-140.

[5] Tougeron, J.Cl., Idéaux de fonctions différentiable, 1. Ann. Inst. Fourier 18, 1 (1968), 177-240. 